\title{
CARACTERIZACIÓN DE POBLACIONES SOBRESALIENTES DE MAÍZ DE LA RAZA ZAPALOTE CHICO
}

\section{CHARACTERIZATION OF OUTSTANDING MAIZE POPULATIONS OF THE ZAPALOTE CHICO RACE}

\section{José M. Cabrera-Toledo*, Aquiles Carballo-Carballo², José A. Mejía-Contreras², Gabino García-De los Santos ${ }^{2}$ y Humberto Vaquera-Huerta ${ }^{2}$}

\author{
'Tecnológico Nacional de México-Comitancillo, Comitancillo, Oaxaca México. ${ }^{2}$ Colegio de Posgraduados, Campus Montecillo, Texcoco, Estado de \\ México, México. \\ *Autor de correspondencia (cabrera.jose@colpos.mx)
}

\section{RESUMEN}

La raza Zapalote Chico de maíz (Zea mays L.) es un componente importante dentro de la diversidad genética de esta especie en la región del Istmo de Tehuantepec, Oaxaca, México. Esta raza suscita interés entre los agricultores locales e investigadores con fines de preservación y difusión. Salvaguardar los recursos fitogenéticos es de relevancia para asegurar la soberanía alimentaria mexicana. Bajo el contexto del cambio climático, las características agronómicas y bondades de Zapalote Chico constituyen una opción viable para considerarse en los programas de mejoramiento nacional e internacional. El objetivo del presente estudio fue realizar una caracterización morfológica de 18 poblaciones nativas de maíz sobresalientes de la raza Zapalote Chico para determinar el grado de variabilidad existente. Se registraron 16 variables cuantitativas y nueve cualitativas de la planta, la espiga y la mazorca. El análisis de varianza sobre las variables cuantitativas mostró que en 13 variables hubo diferencia estadística altamente significativa y en dos la diferencia fue significativa. En el análisis de componentes principales los tres primeros componentes explicaron el $59.06 \%$ de la varianza acumulada; las características con mayor valor descriptivo de la variabilidad fenotípica entre las poblaciones evaluadas fueron la relación entre la altura de la mazorca superior y altura de planta, altura de planta, altura de mazorca, número de hileras de la mazorca, diámetro de mazorca, longitud de espiga y longitud del eje central de la espiga. Las colectas que más contribuyeron a la variabilidad fueron ZAP-MOR, OAX-827, OAX-832 y COL-51. La similitud fue más evidente en las poblaciones de mayor y menor altitud, ya que las de altura intermedia se apartaron notablemente, lo que puede obedecer a que son de la localidad de estudio y se probaron ahí mismo.

Palabras clave: Zea mays, similitud, variabilidad morfológica, variación genética, Zapalote Chico.

\section{SUMMARY}

The Zapalote Chico race of maize (Zea mays L.) is an important component of the genetic diversity of this species in the Tehuantepec Isthmus region, Oaxaca, Mexico. This race raises interest among local farmers and researchers for preservation and dissemination purposes. Safeguarding plant genetic resources is of relevance to ensure Mexican food sovereignty. Under the context of climate change, the agronomic characteristics and virtues of Zapalote Chico constitute a viable option to be considered in national and international breeding programs. The objective of the present study was to perform a morphological characterization of 18 outstanding landraces of the Zapalote Chico race to determine the degree of the extant variability. Sixteen quantitative and nine traits of the plant, tassel and ear were recorded. The analysis of variance of the quantitative traits showed highly significant statistical differences in 13 traits and in two the difference was significant In the principal component analysis the first three components accounted for $59.06 \%$ of the cumulative variance; the characters with the greatest descriptive value of phenotypic variability between the populations evaluated were the ratio between the height of the upper ear and plant height, plant height, ear height, number of kernel rows, ear diameter and tassel central axis length. The accessions that contributed the most to variability were ZAP-MOR OAX-827, OAX-832 and COL-51. Similarity was more evident in populations from higher and lower altitudes, as those from the intermediate altitude were markedly separated, which may be due to the fact that they are from the study location and were tested at the same place.

Index words: Zea mays, similarity, morphological variability, genetic variation, Zapalote Chico.

\section{INTRODUCCIÓN}

La superficie cosechada de maíz (Zea mays L.) en la región del Istmo de Tehuantepec, Oaxaca durante el año 2018 fue de 75,339 ha, con una producción de 110,683 t y un rendimiento promedio de $1.47 \mathrm{t} \mathrm{ha}^{-1}$ (SIAP, 2019) En esta región, predominantemente zapoteca, el maíz se cultiva principalmente bajo condiciones de temporal y con el uso de semilla nativa.

En la actualidad predomina en la región un común interés entre los agricultores locales e investigadores en preservar, fomentar y difundir el uso de las variedades nativas de la raza Zapalote Chico, que a lo largo de su historia han sido cultivadas y aprovechadas en las comunidades de la región Istmeña de Oaxaca; no obstante, los cambios en las formas de consumo, tipificadas en la comida rápida, que en estos días experimentan los habitantes de la zona, en los últimos años se ha agudizado la sensibilidad de preferencia de sectores de la población urbana y rural por productos alimenticios preparados en forma autóctona o tradicional con maíces nativos, tal es el caso de las tortillas y totopos que se elaboran de maíz Zapalote Chico, que se distinguen por su mejor calidad comparada con la harina de maíz híbrido nixtamalizado. 
Investigaciones realizadas en la región del Istmo de Tehuantepec como las de Wellhausen et al. (1951), Muñoz (2005), López-Romero et al. (2005) y Taba et al. (2006) han evidenciado la existencia de una considerable variabilidad en los caracteres agronómicos, entre las poblaciones nativas de esta raza.

Ortega (2003) señala que la importancia de conocer la diversidad nativa del maíz es entender y proteger las relaciones entre el hombre y el maízy de esa forma contribuir al conocimiento científico de esta planta paradigmática a nivel mundial y sobre todo en México. Al Zapalote Chico se le han detectado 22 complejos genéticos favorables, que lo convierte en una de las razas más perfeccionadas del planeta, condición que establece que entre los maíces nativos hay variedades superiores, no sólo en rendimiento, sino en características relacionadas con sus usos. Lo anterior, señala la importancia de la megadiversidad de los maíces nativos (Hernández-Vázquez et al., 2018) como fuente para mejorar otros maíces, no al revés (Muñoz, 2005). A nivel internacional la raza Zapalote Chico ha recibido particular atención por su resistencia a la mosca de seda Euxesta stigmatias Loew. (Diptera: Otitidae), al gusano cogollero Spodoptera frugiperda (J. E. Smith) y al gusano elotero Heliocoverpa zea (Boddie) (Lepidoptera: Noctuidae), razón por la que se registró una población de esta raza en los E.E.U.U. (Widstrom et al., 2003).

Los usos especiales de las variedades nativas, especialmente los usos culinarios específicos, determinan el gran interés por esta raza (Fernández et al., 2013). Debido a la importancia de este grano básico en la región, así como por las evidencias de la diversidad genética existente y los escasos estudios particulares en la caracterización racial se llevó la presente investigación, cuyo objetivo fue determinar el grado de diversidad morfológica que existe en poblaciones sobresalientes de la raza Zapalote Chico del Istmo de Tehuantepec, estado de Oaxaca, México.

\section{MATERIALES Y MÉTODOS}

\section{Germoplasma}

El material biológico consistió en 18 criollos de maíz de grano blanco seleccionados de la raza Zapalote Chico, procedente de la región del Istmo de Tehuantepec, Oaxaca, México, considerada como el área geográfica de distribución de dicha raza (Aragón et al., 2018). Estos materiales se derivaron de una colección regional realizada por investigadores del Instituto Nacional de Investigaciones Forestales, Agrícolas y Pecuarias (INIFAP) y del Instituto Tecnológico de Comitancillo (ITC) en el año de 1997. La depuración de las accesiones se realizó considerando aspectos de identificación de la raza de interés, así como valoración agronómica, de preferencia de productores. En el Cuadro 1 se señalan los lugares de colecta y denominación de los materiales en estudio.

\section{Sitio, diseño y unidad experimental}

Durante el ciclo agrícola PV/2012, en los terrenos del Instituto Tecnológico de Comitancillo, Oaxaca, se estableció un ensayo experimental para el incremento y caracterización de las 18 poblaciones en pequeñas parcelas de producción. La unidad experimental consistió de una planta y fueron seleccionadas 20 de ellas de forma aleatoria a manera de repeticiones (Carballo y Ramírez, 2010).

\section{Manejo agronómico del experimento}

Se usaron parcelas de 19 surcos de $7.5 \mathrm{~m}$ de largo y separados a $0.55 \mathrm{~m}$, con un arreglo de dos semillas por mata a una distancia de $0.5 \mathrm{~m}$, con una densidad de población aproximada de 72,700 plantas por hectárea. La siembra se realizó el 13 de julio de 2012. Se usó la dosis de fertilización 92N-46P-00K (200 kg de urea y 100 kg de superfosfato de calcio triple); en la siembra se aplicó la mitad del nitrógeno y todo el fosforo y el resto del nitrógeno se suministró en el aporque.

\section{Variables evaluadas}

Las variables de estudio se enlistan en el Cuadro 2. En cada parcela se marcaron al azar 20 plantas para el registro de las variables vegetativas y de espiga. Las características de mazorca se determinaron una vez realizada la cosecha sobre 20 mazorcas de cada variedad. La metodología para el registro de las variables evaluadas se basó en el manual gráfico para la descripción varietal de maíz (Carballo y Ramírez, 2010).

\section{Análisis estadísticos}

Con los datos de las 16 variables cuantitativas se aplicó un análisis de varianza bajo un diseño completamente al azar y se utilizó la prueba de comparación de medias de Tukey $(P \leq 0.05)$ Los promedios por población de estas variables fueron estandarizados y con esta información se procedió a practicar un análisis de componentes principales, todo ello utilizando el programa SAS versión 9.1 para Windows (SAS Institute, 2004). A las nueve variables cualitativas únicamente se les determinó el porcentaje de homogeneidad. 
Cuadro 1. Sitios de colecta y denominación de 18 poblaciones de la raza Zapalote Chico utilizadas en el estudio.

\begin{tabular}{lccc}
\hline Colecta & Localidad & Nombre local & Altitud (msnm) \\
\hline OAX-823 & San Pedro Comitancillo & Hoja morada & 76 \\
OAX-826 & San Pedro Comitancillo & Zapalote chico & 72 \\
OAX-827 & San Pedro Comitancillo & Zapalote & 70 \\
OAX-830 & Sto. Dgo Chihuitán & Zapalote blanco & 96 \\
OAX-832 & Sto. Dgo Chihuitán & Cuarentena & 99 \\
OAX-834 & Santiago Laollaga & Zapalote & 127 \\
OAX-838 & Santiago Laollaga & Zapalote & 130 \\
COL-29 & Sn Fco. Ixhuatán & Zapalote & 16 \\
COL-31 & Montecillo Sta. Cruz & Zapalote & 15 \\
COL-32 & Montecillo Sta. Cruz & Zapalote enredado & 10 \\
COL-34 & Unión Hidalgo & Zapalote morado & 15 \\
COL-36 & Unión Hidalgo & Zapalote chico & 17 \\
COL-45 & Sta. Ma. Xadani & Maíz chiquito & 10 \\
COL-51 & Álvaro Obregón & Olote colorado & 25 \\
COL-58 & San Blas Atempa & Maíz chico & 35 \\
COL-62 & El Morro Mazatán & Criollo & 19 \\
COL-64 & El Morro Mazatán & Chiquito morado & 5 \\
ZAP MOR & San Pedro Comitancillo & Zapalote morado & 70 \\
\hline
\end{tabular}

\section{RESULTADOS Y DISCUSIÓN}

\section{Análisis de varianza}

El análisis de varianza indicó que, entre las 18 poblaciones sobresalientes, hubo diferencias altamente significativas en 13 variables y significativas en dos (Cuadro 3). Los indicios de las diferencias estadísticas entre los maíces nativos evaluados, demuestran la diversidad genética existente en la raza Zapalote Chico. Esta variación se atribuye a las prácticas de selección que el productor ha hecho para tipo de grano, forma y tamaño de mazorca, número de hileras, grosor de olote y precocidad, entre otros criterios. Los datos de las variables estudiadas concuerdan con los estudios realizados por López-Romero et al. (2005), Muñoz (2005) y Taba et al. (2006), quienes describen que en la región del Istmo de Tehuantepec, Oaxaca se presenta variación entre razas y dentro de las poblaciones de la raza Zapalote Chico en varias características contrario a la percepción que se tenía de poca variación en el maíz Zapalote Chico.

\section{Características agronómicas}

La variación de los caracteres agronómicos evaluados (vegetativo, espiga y mazorca) de las 18 poblaciones sobresalientes se muestra en el Cuadro 4.

En el Cuadro 4 se muestran las medias de las poblaciones estudiadas para las 16 variables cuantitativas Algunos aspectos a resaltar son que la variedad OAX-834 tuvo la mayor precocidad en floración masculina junto un grupo de 10 variedades que la igualaron estadísticamente, lo cual es importante, pues se trata de un carácter muy apreciado en la región, ya que es común que este tipo de maíz se cultive en el mismo terreno por más de un ciclo al año. La variedad ZAP-MOR junto con algunas otras fueron superiores en DFM y DFF presentan las cifras más altas en días en ambas floraciones lo que frecuentemente se relaciona con mayor altura de planta y constituye una desventaja para la región de interés, que se caracteriza por los fuertes vientos durante los meses de octubre a marzo, pues una mayor altura en la planta constituye un factor de riesgo para el acame. 
Cuadro 2. Variables registradas en las 18 poblaciones del estudio San Pedro Comitancillo. Oax. PV/2012.

\begin{tabular}{|c|c|c|c|c|}
\hline $\begin{array}{l}\text { Tipo de } \\
\text { Variable }\end{array}$ & Denominación & Abreviatura & $\begin{array}{l}\text { Unidad de } \\
\text { medida }\end{array}$ & $\begin{array}{c}\text { Tamaño de } \\
\text { Muestra }\end{array}$ \\
\hline & Variables cuantitativas & & & \\
\hline \multirow[t]{6}{*}{ Vegetativa } & Días a floración masculina & DFM & días & 20 plantas \\
\hline & Días a floración femenina & DFF & días & 20 plantas \\
\hline & Altura de planta & ALP & $\mathrm{cm}$ & 20 plantas \\
\hline & Altura de mazorca & ALM & $\mathrm{cm}$ & 20 plantas \\
\hline & Relación entre ALM superior y ALP & $\mathrm{RAM} / \mathrm{P}$ & adimensional & 20 plantas \\
\hline & Ancho de lámina & ANL & $\mathrm{cm}$ & 20 plantas \\
\hline \multirow[t]{6}{*}{ Espiga } & Longitud de pedúnculo & LOP & $\mathrm{cm}$ & 20 plantas \\
\hline & Longitud de la espiga & LOE & $\mathrm{cm}$ & 20 plantas \\
\hline & Longitud del eje central de la espiga & LEEP & $\mathrm{cm}$ & 20 plantas \\
\hline & Número de ramas laterales primarias de la espiga & NRELP & número & 20 plantas \\
\hline & Ramas secundarias de la espiga & RES & número & 20 plantas \\
\hline & Longitud de ramas laterales de la espiga & LERL & $\mathrm{cm}$ & 20 plantas \\
\hline \multirow[t]{14}{*}{ Mazorca } & Largo de mazorca & LAM & $\mathrm{cm}$ & 20 mazorcas \\
\hline & Diámetro de mazorca & DIA & $\mathrm{cm}$ & 20 mazorcas \\
\hline & Número de hileras de grano & NHG & número & 20 mazorcas \\
\hline & Número de granos por hilera & $\mathrm{NGH}$ & número & 20 mazorcas \\
\hline & Variables cualitativas & & & \\
\hline & Forma de mazorca & FMA & & 20 mazorcas \\
\hline & Arreglo de hileras & $\mathrm{AHI}$ & & 20 mazorcas \\
\hline & Tipo de grano & TGR & & 20 mazorcas \\
\hline & Forma de la corona de grano & FCG & & 20 mazorcas \\
\hline & Color de grano & CGR & & 20 mazorcas \\
\hline & Color dorsal del grano & $C D G$ & & 20 mazorcas \\
\hline & Color del endospermo del grano & CEG & & 20 mazorcas \\
\hline & Coloración por antocianina en las glumas & CAG & & 20 mazorcas \\
\hline & Intensidad de coloración por antocianina en las glumas & ICAG & & 20 mazorcas \\
\hline
\end{tabular}

En las características de espiga, sobresale la variedad OAX-827 y las del mismo grupo estadístico, cuyos valores en longitud de espiga y longitud de ramas laterales de la espiga fueron las más altas. Estas características pueden ser ventajosas, al tener una mayor amplitud para la producción de polen, lo cual es importante debido a que en condiciones de viento éste se dispersa en una mayor superficie y por lo tanto se requieren mayores cantidades para una polinización efectiva.

Las mejores características de mazorca se observaron en la variedad OAX-838 y las del mismo grupo estadístico, que fue además uno de los materiales más tardíos en floración femenina. La variedad ZAP-MOR tuvo los mejores valores en diámetro de mazorca, y en longitud de mazorca presentó el menor valor; estos caracteres ubican a esta variedad, por sus características de mazorca, como semejantes a los de la raza Pepitilla. El número de hileras de grano que presentó el ZAP-MOR fue superior a lo encontrado por Martin et al. (2008) en colectas de la raza Pepitilla. ZAP-MOR es un genotipo que, en la región del Istmo de Tehuantepec, está teniendo aceptación por los productores, ya que produce gran volumen de granos y por su abundante número de hileras de granos, además, la coloración del tallo, espiga, totomoxtle y olote se presenta en diferentes intensidades de color morado (Cuadro 5), como resultado a la selección por parte de los productores.

Los elementos vertidos confirman que el cultivo de maíz es un sistema continuo y dinámico, su polinización abierta 
Cuadro 3. Estadísticos de 16 variables cuantitativas, evaluadas las 18 del estudio. San Pedro Comitancillo, Oax. PV/2012.

\begin{tabular}{|c|c|c|c|c|}
\hline \multirow{2}{*}{ Variable } & \multicolumn{2}{|c|}{ Cuadrados medios } & \multirow{2}{*}{ Media } & \multirow{2}{*}{ CV $(\%)$} \\
\hline & Variedades & Error & & \\
\hline Días a floración masculina & $17.88 * *$ & 3.46 & 47.43 & 3.92 \\
\hline Longitud del pedúnculo & $22.77 \mathrm{~ns}$ & 23.55 & 9.41 & 51.55 \\
\hline Longitud de espiga & $59.16 * \star$ & 17.78 & 33.15 & 12.72 \\
\hline Longitud del eje central de la espiga & $22.29 *$ & 13.15 & 23.59 & 15.37 \\
\hline Número de ramas laterales primarias de la espiga & $38.30 * *$ & 11.39 & 10.9 & 30.97 \\
\hline Ramas secundarias de la espiga & $2.71 *$ & 1.51 & 1.84 & 66.84 \\
\hline Días a floración femenina & $17.19 * *$ & 4.99 & 49.18 & 4.54 \\
\hline Longitud de ramas laterales de la espiga & $64.53 * *$ & 15.02 & 20.28 & 19.11 \\
\hline Altura de planta & $1414.25 * *$ & 389.93 & 196.97 & 10.03 \\
\hline Altura de la mazorca & $1240.47 * *$ & 197.41 & 79.64 & 17.64 \\
\hline Relación entre ALM superior y ALP & $0.022 * \star$ & 0.004 & 0.4 & 15.36 \\
\hline Ancho de lámina & $2.67 * *$ & 0.914 & 6.42 & 14.89 \\
\hline Largo de mazorca & $25.97 \star \star$ & 1.62 & 11.32 & 11.25 \\
\hline Diámetro de mazorca & $0.518 * *$ & 0.079 & 3.99 & 7.05 \\
\hline Numero de hileras de grano & $43.61 * *$ & 2.12 & 11.15 & 13.06 \\
\hline Numero de granos por hilera & $54.68 * *$ & 11.7 & 25.61 & 13.36 \\
\hline
\end{tabular}

*: significancia al $5 \%$, **: significancia al $1 \%$, ns: no significativo, CV: coeficiente de variación.

implica un constantemovimiento de genes y los agricultores nativos en forma constante realizan un movimiento o flujo de la semilla que mantienen, intercambian y experimentan. La frecuencia de recombinación genética es tan dinámica que es posible un alto número de combinaciones, lo que permite a los agricultores contar con nuevas características y atributos de este cultivo por medio de la selección (Marielle et al., 2013)

\section{Análisis de componentes principales}

Para una mejor interpretación de la información obtenida, se utilizó la técnica de análisis multivariado de componentes principales (ACP), aplicado a los promedios por variedad para las 16 variables cuantitativas en estudio. Este análisis es muy útil para discriminar variables, así como seleccionar los genotipos con mejores atributos y considerar el conjunto de variables en su totalidad (Johnson y Wichern, 1992).

Los porcentajes de la varianza explicados por cada componente, se presentan en el Cuadro 6. Se analizaron 16 variables cuantitativas que arrojaron igual número de componentes principales, donde los primeros tres aportaron $59.06 \%$ de la varianza explicada.

Las variables más importantes en la definición del primer componente principal con base en los coeficientes de los vectores propios fueron seis, mientras que las más importantes en la definición del segundo componente principal fueron cuatro (Cuadro 7). Hortelano et al. (2008), al estudiar los maíces nativos del Valle de Puebla, señalan el diámetro de mazorca entre las variables de mayor valor discriminativo, lo que puede ser razonable dada la mayor amplitud de los tipos de maíz que ellos usaron.

Para el tercer componente, fueron los días a floración masculina y el número de hileras de grano, las características de mayor contribución con signos negativos -4617 y -0.3797 , respectivamente. Al respecto existe coincidencia con lo encontrado por Martin et al. (2008), cuando caracterizaron maíces nativos del noroccidente de México, donde la variable días a floración masculina fue importante para el segundo componente (-0.6370). Con valor positivo aparece el ancho de lámina (0.3610), que es un componente de disminución de área foliar" condicionado por la precocidad que identifica a los materiales genéticos de la raza en estudio.

En la contribución relativa de cada variedad para los componentes principales en estudio, sobresalen las cuatro variedades: ZAP-MOR, OAX-827, OAX-832 y COL-51 (Cuadro 8). Al ubicar los materiales en un plano determinado por los primeros componentes principales (Figura 1) y tomando en cuenta la matriz de correlaciones, los materiales procedentes de San Pedro Comitancillo, 


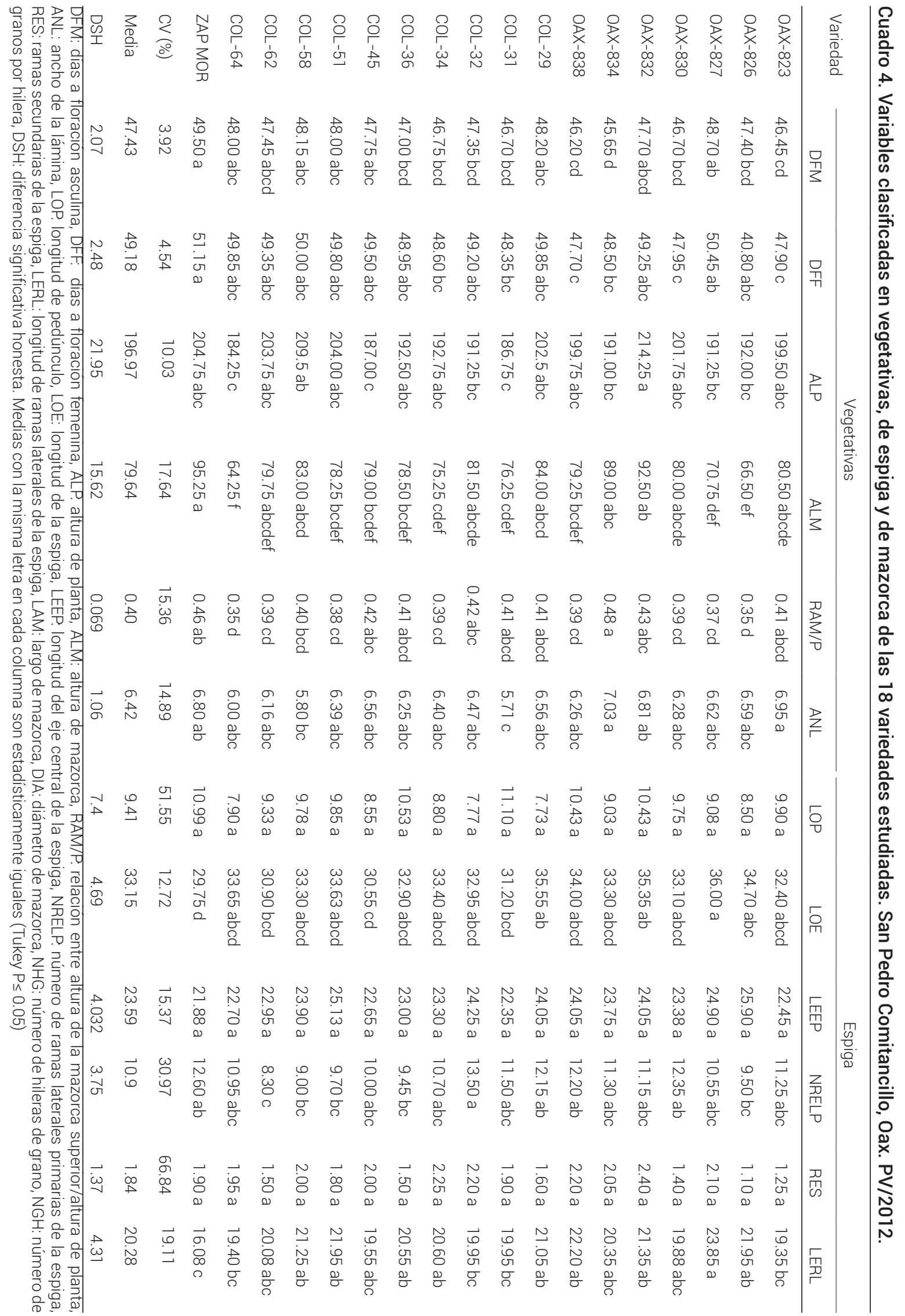


Cuadro 4. Cotinuación

\begin{tabular}{clll}
\hline \multicolumn{4}{c}{ Mazorca } \\
\hline \multicolumn{1}{c}{ LAM } & \multicolumn{1}{c}{ DIA } & \multicolumn{1}{c}{ NHG } & \multicolumn{1}{c}{ NGH } \\
\hline $9.82 \mathrm{fgh}$ & $3.74 \mathrm{~d}$ & $9.90 \mathrm{~d}$ & $25.35 \mathrm{abcde}$ \\
$12.18 \mathrm{abcd}$ & $3.84 \mathrm{~cd}$ & $10.30 \mathrm{~d}$ & $25.50 \mathrm{abcde}$ \\
$12.76 \mathrm{ab}$ & $4.24 \mathrm{ab}$ & $11.30 \mathrm{bcd}$ & $26.96 \mathrm{abcd}$ \\
$12.00 \mathrm{abcd}$ & $4.07 \mathrm{abc}$ & $11.5 \mathrm{bcd}$ & $28.50 \mathrm{a}$ \\
$10.36 \mathrm{efgh}$ & $3.91 \mathrm{~cd}$ & $10.00 \mathrm{~d}$ & $24.55 \mathrm{bcde}$ \\
$10.97 \mathrm{cdefg}$ & $3.94 \mathrm{bcd}$ & $10.20 \mathrm{~d}$ & $26.45 \mathrm{abcde}$ \\
$13.30 \mathrm{a}$ & $4.13 \mathrm{abc}$ & $11.40 \mathrm{bcd}$ & $28.20 \mathrm{ab}$ \\
$10.82 \mathrm{defg}$ & $4.13 \mathrm{abc}$ & $10.60 \mathrm{bcd}$ & $26.70 \mathrm{abcde}$ \\
$9.68 \mathrm{gh}$ & $3.84 \mathrm{~cd}$ & $10.80 \mathrm{bcd}$ & $24.55 \mathrm{bcde}$ \\
$10.05 \mathrm{fgh}$ & $3.96 \mathrm{bcd}$ & $10.50 \mathrm{~cd}$ & $23.00 \mathrm{~d}$ \\
$10.91 \mathrm{cdefg}$ & $3.90 \mathrm{~cd}$ & $10.40 \mathrm{~cd}$ & $26.35 \mathrm{abcde}$ \\
$12.24 \mathrm{abc}$ & $3.91 \mathrm{~cd}$ & $10.50 \mathrm{~cd}$ & $25.25 \mathrm{abcde}$ \\
$11.72 \mathrm{bcde}$ & $4.14 \mathrm{abc}$ & $12.20 \mathrm{~b}$ & $27.20 \mathrm{abc}$ \\
$12.00 \mathrm{abcd}$ & $3.96 \mathrm{bcd}$ & $11.30 \mathrm{bcd}$ & $24.95 \mathrm{abcde}$ \\
$12.31 \mathrm{abc}$ & $3.89 \mathrm{~cd}$ & $10.40 \mathrm{~cd}$ & $26.90 \mathrm{abcd}$ \\
$12.07 \mathrm{abcd}$ & $4.03 \mathrm{bcd}$ & $12.00 \mathrm{bc}$ & $23.20 \mathrm{ed}$ \\
$11.21 \mathrm{cdef}$ & $3.84 \mathrm{~cd}$ & $11.00 \mathrm{bcd}$ & $24.00 \mathrm{cde}$ \\
$9.37 \mathrm{~h}$ & $4.37 \mathrm{a}$ & $16.45 \mathrm{a}$ & $23.40 \mathrm{cde}$ \\
11.25 & 7.05 & 13.06 & 13.36 \\
11.32 & 3.99 & 11.15 & 25.61 \\
1.42 & 0.31 & 1.62 & 3.8 \\
\hline
\end{tabular}

Oaxaca (OAX-823, OAX-826, OAX-827 y ZAP-MOR) se encuentran representados en los cuatro cuadrantes, dos que se separan hacia los extremos de la figura (Grupos 3 y 4 ) y los otros dos incluidos en los agrupamientos centrales (Grupos 1 y 2). En esta región el maíz de tierras altas se adaptó a las condiciones ambientales de la planicie costera mediante procesos de selección por parte de los primeros agricultores (Muñoz, 2005).

Los materiales del Grupo 3 se destacan en características vegetativas como: floración tardía, mayor porte de planta y mazorca, superiores en ancho de lámina; en mazorca presentan diferencias muy marcadas en longitud, diámetro y número de hileras de granos. En el Grupo 1 se ubicaron preferentemente materiales que se colectaron en localidades de baja altitud.

Se distingue la integración de otro grupo, donde predominaron las variedades cuya procedencia son de localidades demayoraltura, los cuales presentaroncifras bajas de signo positivo en el componente uno, y en el componente dos, cifras altas positivas (Grupo 2). Los materiales de este grupo mostraron características vegetativas sobresalientes en: longitud de espiga, longitud del eje central de la espiga, altura de planta y de mazorca, y diámetro de mazorca.

Adicionalmente, al tomar en cuenta a los 10 sitios de procedencia de las 18 poblaciones en estudio (Figura 2), esta agrupación presenta cierta semejanza con la distribución que se señala en los componentes principales, por lo que es posible identificar los grupos: Zapalotes de altura, Zapalotes de planicie y Zapalotes intermedios.

\section{Análisis de agrupamiento}

Para definir relaciones de similitud más precisa entre las 18 poblaciones, se generó un dendrograma (Figura 3) con la base en las 16 variables estudiadas. A una distancia de corte de 55.7 al explorar la asociación entre las poblaciones se identificaron tres grupos.

De acuerdo con los valores promedio a nivel de grupo de las 16 variables cuantitativas, las variedades del Grupo 1 sobresalieron en longitud de espiga $(33.91 \mathrm{~cm})$, longitud del eje central de la espiga $(23.71 \mathrm{~cm})$ y ramas secundarias de la espiga (1.9). El Grupo 2 resultó el más numeroso y con características superiores de: longitud de ramas laterales de la espiga (20.93), longitud de mazorca $(11.87 \mathrm{~cm})$ y número de granos por hilera (25.96).

El Grupo 3 representado por una sola variedad (ZAP MOR), presentó mayor valor en días a floración masculina (49.5), longitud del pedúnculo $(10.99 \mathrm{~cm})$, número de ramas laterales primarias de la espiga (12.6), ramas secundarias de la espiga (1.9), días a floración femenina (51.15), altura de planta $(204.75 \mathrm{~cm})$, altura de mazorca $(95.25 \mathrm{~cm})$, relación entre altura de la mazorca superior y altura de planta (0.46), ancho de lámina (6.8), diámetro de mazorca $(4.37 \mathrm{~cm})$ y número de hileras de granos (16).

\section{CONCLUSIONES}

Existe alta variabilidad en las características agronómicas de los maíces de la raza Zapalote Chico de la región Istmeña de Oaxaca. Existe similitud entre las poblaciones de diferente altitud, y ésta fue más evidente en las poblaciones de mayor y menor altitud. Las características con mayor valor descriptivo de la variabilidad fenotípica entre las poblaciones evaluadas fueron la: relación entre la altura de mazorca superior y altura de planta, altura de planta, altura de mazorca y número de hileras de grano, diámetro de mazorca, longitud de espiga y longitud del eje central de la espiga. 
Cuadro 5. Porcentaje de homogeneidad de variables cualitativas de 18 poblaciones sobresalientes de la Raza Zapalote Chico.

\begin{tabular}{|c|c|c|c|c|c|c|}
\hline Variedad & FM & $\mathrm{AH}$ & $\mathrm{TG}$ & FCG & CAGO & ICAGO \\
\hline OAX-827 & $\begin{array}{l}\text { Cilíndrica:85 } \\
\text { Cónica cilíndrica: } 15\end{array}$ & Recta: 100 & Intermedio: 100 & Hendida: 100 & $\begin{array}{l}\text { Ausente: } 85 \\
\text { Presente: } 15\end{array}$ & Ausente: 100 \\
\hline OAX-832 & $\begin{array}{l}\text { Cilíndrica: } 90 \\
\text { Cónica cilíndrica: } 10\end{array}$ & Recta: 100 & Intermedio: 100 & Hendida: 100 & $\begin{array}{l}\text { Ausente: } 90 \\
\text { Presente: } 10\end{array}$ & $\begin{array}{l}\text { Ausente: } 90 \\
\text { Media: } 10\end{array}$ \\
\hline OAX-834 & Cilíndrica: 100 & Recta: 100 & Intermedio: 100 & Hendida: 100 & Ausente: 100 & Ausente: 100 \\
\hline COL-31 & $\begin{array}{l}\text { Cilíndrica: } 90 \\
\text { Cónica cilíndrica: } 10\end{array}$ & Recta: 100 & Intermedio: 100 & Hendida: 100 & Ausente: 95 & $\begin{array}{l}\text { Ausente: } \\
10\end{array}$ \\
\hline COL-32 & Cilíndrica 100 & Recta: 100 & Intermedio: 100 & Hendida: 100 & $\begin{array}{l}\text { Ausente: } 60 \\
\text { Presente: } 40\end{array}$ & $\begin{array}{l}\text { Ausente: } 70 \\
\text { Media: } 30\end{array}$ \\
\hline COL-34 & $\begin{array}{l}\text { Cilíndrica: } 90 \\
\text { Cónica cilíndrica: } 10\end{array}$ & Recta: 100 & Intermedio: 100 & Hendida: 100 & $\begin{array}{l}\text { Ausente: } 65 \\
\text { Presente: } 35\end{array}$ & Ausente: 100 \\
\hline COL-36 & $\begin{array}{l}\text { Cilíndrica: } 90 \\
\text { Cónica cilíndrica: } 10\end{array}$ & Recta: 100 & Intermedio: 100 & Hendida: 100 & Ausente: 100 & Ausente: 100 \\
\hline COL-64 & Cilíndrica: 100 & Recta: 100 & Intermedio: 100 & Hendida: 100 & $\begin{array}{l}\text { Ausente: } 80 \\
\text { Presente: } 20\end{array}$ & Ausente: 100 \\
\hline ZAP-MOR & $\begin{array}{l}\text { Cilíndrica } 85 \\
\text { Cónica cilíndrica: } 15\end{array}$ & $\begin{array}{l}\text { Recta: } 85 \\
\text { Espiral: } 15\end{array}$ & $\begin{array}{l}\text { Intermedio: } \\
100 \%\end{array}$ & Hendida: 100 & $\begin{array}{l}\text { Ausente: } 15 \\
\text { Presente: } 85\end{array}$ & $\begin{array}{l}\text { Ausente: } 85 \\
\text { Media: } 15\end{array}$ \\
\hline
\end{tabular}

FM: forma de mazorca, AH: arreglo de hileras, TG: tipo de grano, FCG: forma de la corona del grano, CAGO: coloración por antocianina en las glumas del olote, ICAGO: intensidad de coloración por antocianina en las glumas del olote. 
Cuadro 6. Análisis de componentes principales en las variables derivadas de 16 caracteres morfológicos. San Pedro Comitancillo, Oax., PV/2012.

\begin{tabular}{lccc}
\hline Componente & Valor propio & $\begin{array}{c}\text { Varianza } \\
\text { total (\%) }\end{array}$ & $\begin{array}{c}\text { Varianza } \\
\text { acumulada (\%) }\end{array}$ \\
\hline 1 & 4.97 & 31.06 & 31.06 \\
2 & 2.40 & 15.02 & 46.08 \\
3 & 2.08 & 12.98 & 59.06 \\
\hline
\end{tabular}

Cuadro 7. Vectores propio y la contribución relativa de cada variable en tres componentes principales derivadas de 18 poblaciones de maíz de la raza Zapalote Chico. San Pedro Comitancillo, Oax., PV/2012.

\begin{tabular}{lccc}
\hline Variable & \multicolumn{3}{c}{ Componentes principales } \\
\cline { 2 - 4 } original & Comp. 1 & Comp. 2 & Comp. 3 \\
\hline DFM & 0.1270 & 0.2209 & -0.4617 \\
LOP & 0.1764 & 0.0151 & -0.0281 \\
LOE & -0.3047 & 0.3409 & 0.1750 \\
LEEP & -0.3207 & 0.3080 & 0.0663 \\
NRELP & 0.1837 & 0.1062 & 0.2912 \\
RES & 0.1023 & 0.2785 & 0.0752 \\
DFF & 0.2578 & 0.1993 & -0.1749 \\
LERL & -0.3712 & 0.2891 & 0.0257 \\
APL & 0.1061 & 0.3453 & 0.0204 \\
ALM & 0.3363 & 0.2651 & 0.2504 \\
RAM/P & 0.3364 & 0.1060 & 0.3455 \\
ANL & 0.1051 & 0.2224 & 0.3610 \\
LAM & -0.2983 & 0.2139 & -0.2789 \\
DIA & 0.1966 & 0.3943 & -0.3002 \\
NHG & 0.3202 & 0.0841 & -0.3797 \\
NGH & -0.1732 & 0.2633 & 0.0666 \\
\hline
\end{tabular}

\section{BIBLIOGRAFÍA}

Aragón C. F. (2018) Actualización de la información sobre los maíces criollos de Oaxaca. Versión 1.5. Comisión Nacional para el Conocimiento y Uso de la Biodiversidad. México D.F https://doi. org/10.15468/rnd0ec

Carballo C. A. y M. E. Ramírez (2010) Manual Gráfico para la Descripción Varietal en Maíz (Zea mays L.). $2^{\text {a }}$ edición Colegio de Postgraduados-Servicio Nacional de Inspección y Comercio de Semillas. Montecillo, Texcoco, México. 68 p.

Fernández S. R., L. A. Morales C. y A. Gálvez M. (2013) Importancia de los maíces nativos de México en la dieta nacional. Una revisión indispensable. Revista Fitotecnia Mexicana 36:275-283.

Hernández-Vázquez B., M. C. Mendoza-Castillo, F. Castillo-González, J. A. Pecina-Martínez, A. Delgado-Alvarado, R. Lobato-Ortiz y J. J. García Zavala (2018) Valoración agromorfólogica de germoplasma de maíz amarillo en Valles Altos de México. Revista Fitotecnia Mexicana 41:393-402.
Cuadro 8. Ubicación de las variedades de acuerdo con los dos primeros componentes principales.

\begin{tabular}{lcc}
\hline \multirow{2}{*}{ Variedades } & \multicolumn{2}{c}{ Componentes } \\
\cline { 2 - 3 } OAX-823 & 0.6112 & -1.9480 \\
OAX-826 & -4.4908 & -1.1676 \\
OAX-827 & -2.2451 & 2.7407 \\
OAX-830 & -0.2562 & 0.2285 \\
OAX-832 & 0.8949 & 2.1306 \\
OAX-834 & 0.7580 & 0.3624 \\
OAX-838 & -1.2565 & 1.7009 \\
COL-29 & -0.2029 & 1.7382 \\
COL-31 & 0.9534 & -2.758 \\
COL-32 & 0.8000 & -0.3388 \\
COL-34 & -0.7240 & -0.5129 \\
COL-36 & -0.4499 & -1.0803 \\
COL-45 & 1.0779 & -0.3253 \\
COL-51 & -1.1123 & 1.0119 \\
COL-58 & -0.7842 & 0.8480 \\
COL-62 & 0.4811 & -1.1345 \\
COL-64 & -1.0672 & -2.1888 \\
ZAP MOR & 7.0126 & 0.6932 \\
\hline
\end{tabular}

Hortelano S. R. R., A. Gil M., A. Santacruz V., S. Miranda C. y L. Córdova T. (2008) Diversidad morfológica de maíces nativos del Valle de Puebla. Agricultura Técnica en México 34:189-200.

Johnson R. A. and D. W. Wichern. (1992) Applied Multivariate Statistical Analysis. Third Edition. Prentice-Hall, Englewood Cliffs. New Jersey. 642 p.

López-Romero G., A. Santacruz V., A. Muñoz-Orozco, F. Castillo- González, L. Córdova-Téllez H. Vaquera-Huerta (2005) Caracterización morfológica de poblaciones nativas de maíz del Istmo de Tehuantepec, México. Interciencia 30:284-290.

Marielle C., A. Turrent F., L. Díaz M. Astier, N. Barrera-Bassol, C. H. Ávila B. y A. C. Dolores F. (2013) Alternativas tecnológicas no transgénicas para el mejoramiento y la producción sustentable de maíz en México. In: El Maíz en Peligro ante los Transgénicos. Un Análisis Integral sobre el Caso de México. E. R. Álvarez-Buylla y A. Piñeyro N. (coords). UNAM-Centro de Investigaciones Interdisciplinarias en Ciencias y Humanidades-Unión de Científicos Comprometidos con la Sociedad-Universidad Veracruzana. México. D. F. pp:333-375.

Martin L. J. G., J. Ron P., J. J. Sánchez G., L. De la Cruz L., M. M. Morales R., J. A. Carrera V., A. Ortega C., V. A. Vidal M. y M. J. Guerrero H. (2008) Caracterización agronómica y morfológica de maíces nativos del noroccidente de México. Revista Fitotecnia Mexicana $31: 331-340$

Muñoz O. A. (2005) Centli-Maíz. Prehistoria e Historia, Diversidad, Potencial, Origen Genético y Geográfico, $2^{\text {a }}$ edición. Colegio de Postgraduados. Montecillo, Estado de México. 210 p

Ortega P. R. (2003) La diversidad del maíz en México. In: Sin Maíz no Hay País. G. Esteva y C. Marielle (coords.). Culturas Populares de México. México D. F. pp:123-154

SIAP, Servicio de Información Agroalimentaria y Pesquera (2019) Anuario estadístico de la producción agrícola 2019. Servicio de Información Agroalimentaria y Pesquera de la Secretaría de Agricultura, Ganadería, Desarrollo Rural, Pesca y Alimentación. 


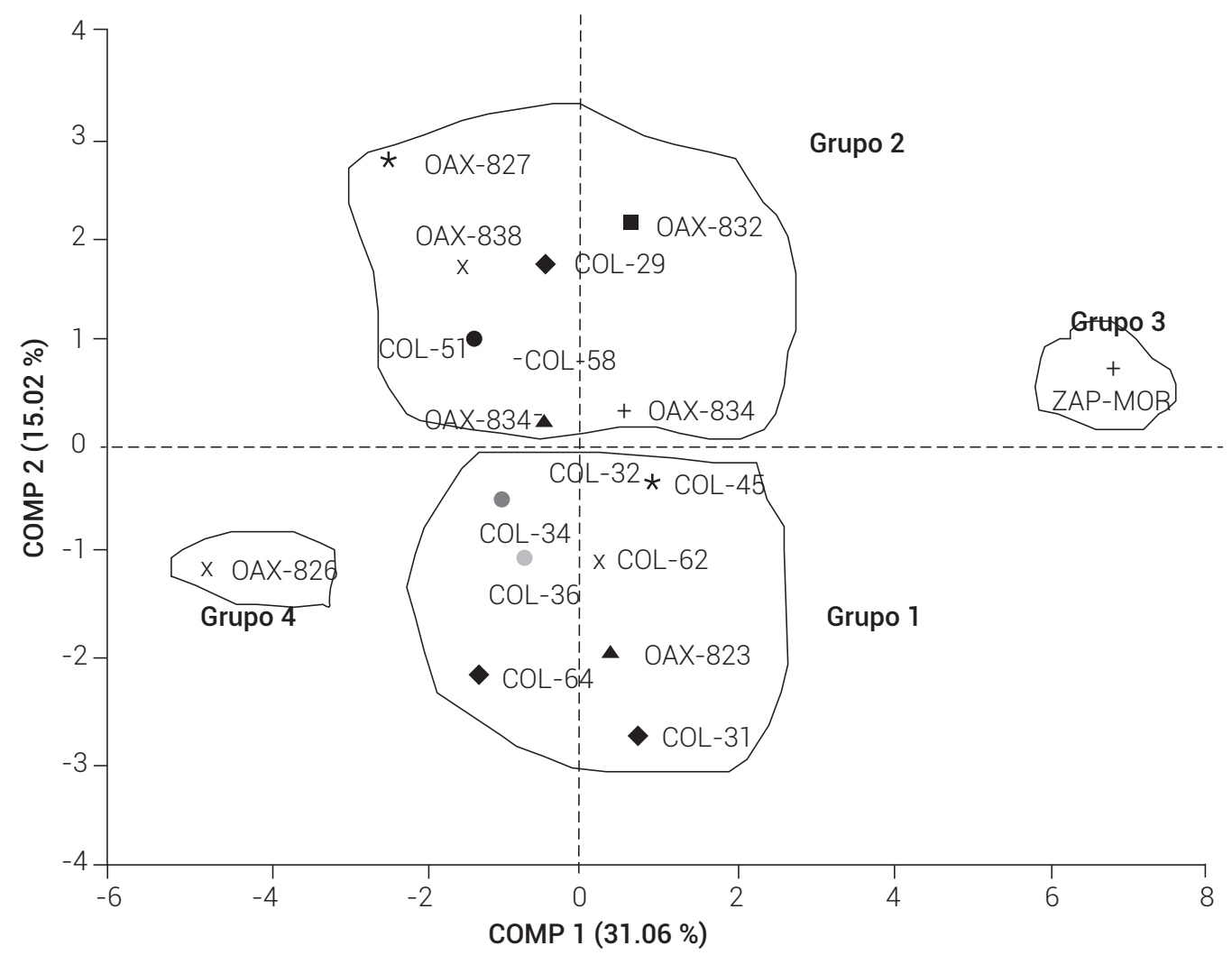

Figura 1. Distribución de 18 poblaciones sobresalientes en relación con los dos primeros componentes principales.

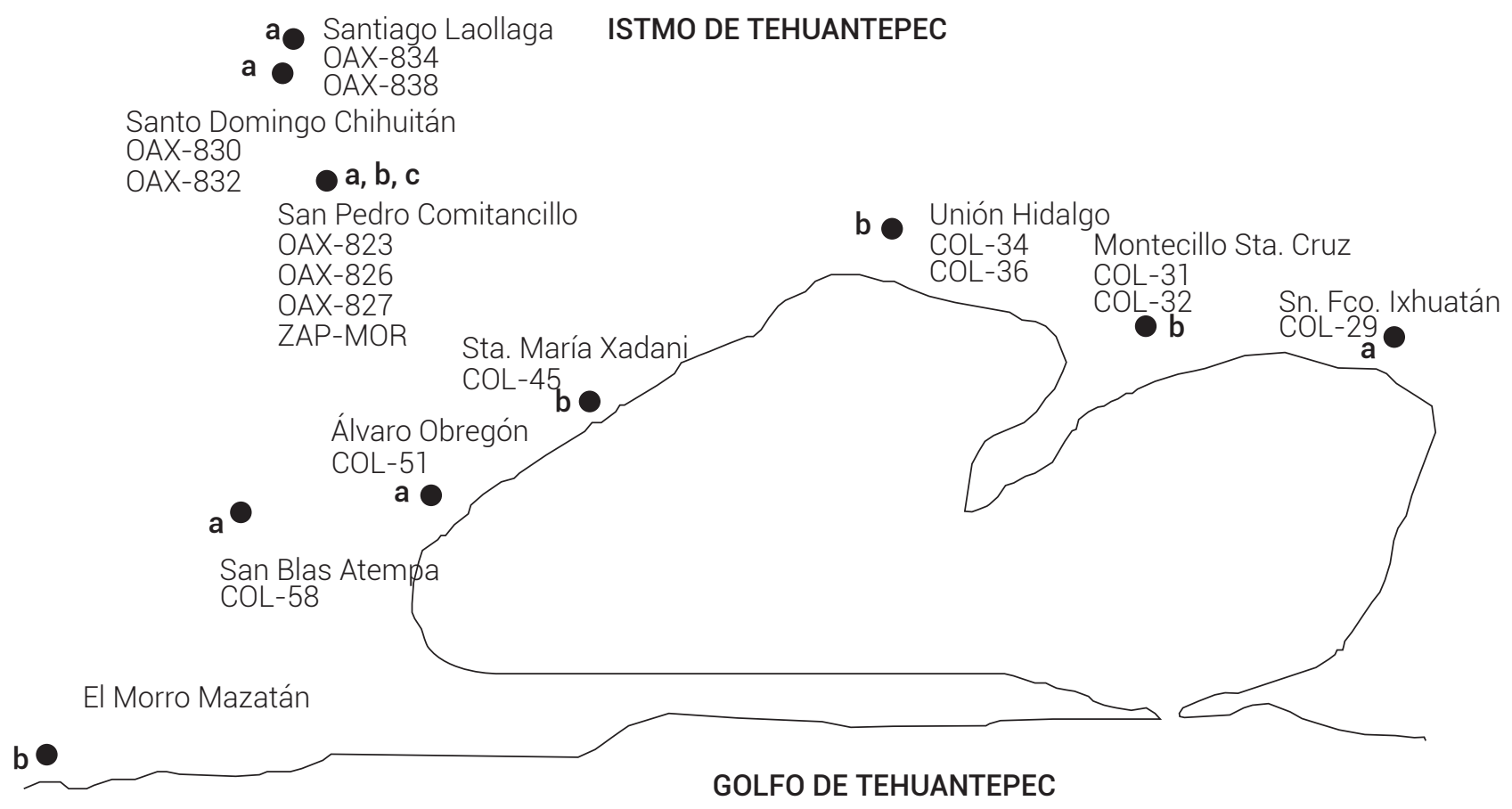

Figura 2. Sitios de origen de los materiales genéticos en estudio y asignación de grupos. a: Zapalote de altura, b: Zapalote de planicie, c: Zapalote intermedio. 


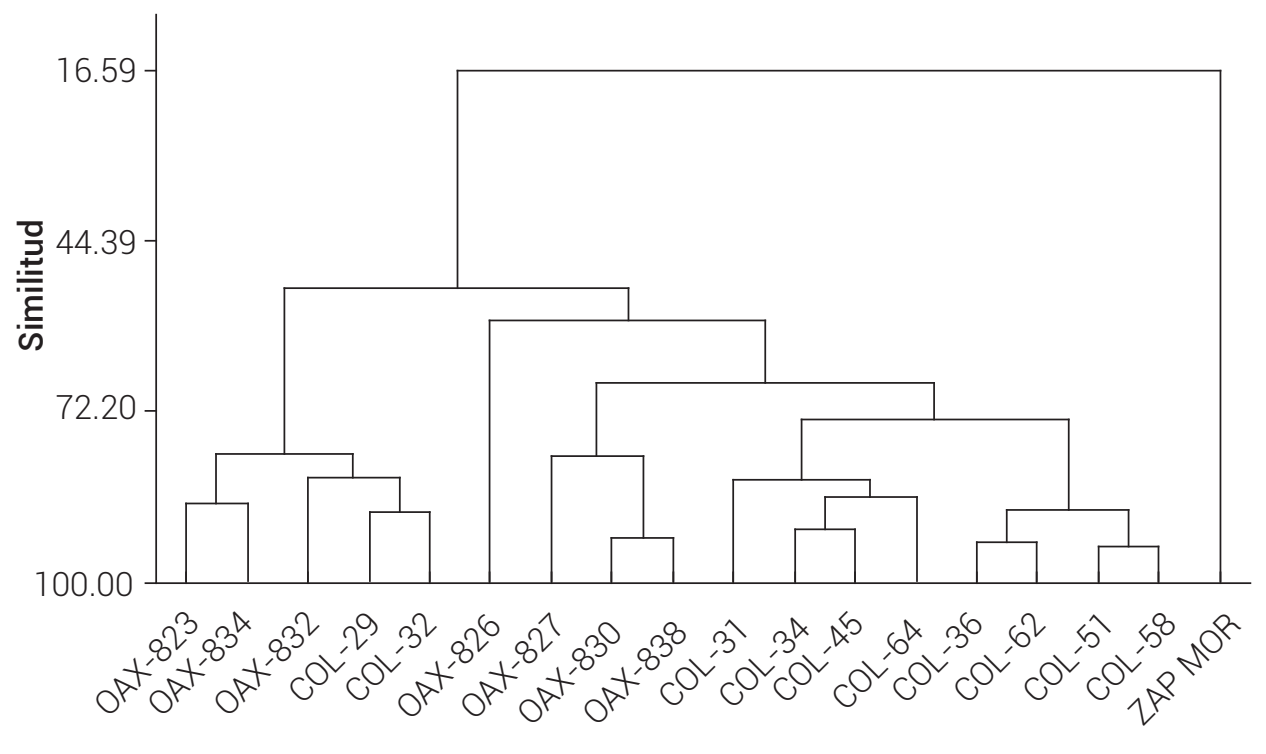

Poblaciones

Figura 3. Dendrograma de 18 poblaciones sobresalientes obtenido a partir de 16 variables por el método de agrupamiento UPGMA.

Ciudad de México. https://nube.siap.gob.mx/cierreagricola (Julio/2019).

SAS, Statistical Analysis System Institute (2004) SAS/STAT® 9.1 User's Guide. SAS Institute Inc., Cary, NC, USA. 4420 p.

Taba S., J. Díaz, F. Aragón C., F. Rincón-Sanchez, J. M. Hernández and M. Krakowsky (2006) Evaluation of Zapalote Chico accessions for conservation and enhancement. Maydica 51:209-218.

Wellhausen E.J., L. M. Roberts, E. Hernández X. y P. C. Mangelsdorf (1951) Razas de Maíz en México, su Origen, Características y
Distribución. Secretaria de Agricultura y Ganadería y Fundación Rockefeller. México. D. F. 239 p.

Widstrom N.W., B. R. Wiseman, M. E. Snook, G. S. Nussly and B. T. Scully (2003) Registration of the maize population Zapalote Chico 2451F. Crop Science 43:444-445. https://doi.org/10.2135/ cropsci2003.444. 
